Opuscula Mathematica • Vol. $30 \bullet$ No. $3 \bullet 2010$

http://dx.doi.org/10.7494/OpMath.2010.30.3.255

\title{
ON THE APPROXIMATION THEOREM OF WONG-ZAKAI TYPE FOR THE LASOTA OPERATOR
}

\author{
Antoni Leon Dawidowicz, Krystyna Twardowska
}

\begin{abstract}
We consider in this paper a stochastic evolution equation with Professor A. Lasota's operator as the infinitesimal generator of a strongly continuous semigroup of transformations and with Hammerstein operator connected with a noise being the Wiener process. We show that such evolution equation satisfies the Wong-Zakai type approximation theorem. The idea of the definition of the Lasota operator has the origin in the mathematical model of the creation and differentiation of cells in biology and medicine.
\end{abstract}

Keywords: stochastic evolution equations, Wong-Zakai approximations, Lasota operator.

Mathematics Subject Classification: Primary: 60H20, 37A10 Secondary: 60H10, $60 \mathrm{H} 25,35 \mathrm{~A} 08$.

\section{INTRODUCTION}

We shall show that a stochastic evolution equation with Professor A. Lasota's operator as the infinitesimal generator of a strongly continuous semigroup of transformations and with Hammerstein operator connected with a noise being the Wiener process satisfies the Wong-Zakai type approximation theorem. The original investigations of the Lasota operator were created in the common paper of A. Lasota and J. Yorke [9] and then by A. Lasota in paper [8]. In the paper [8], there is a new sufficient condition for the existence of the continuous invariant and ergodic measures of the turbulent trajectories for some semi-dynamical systems in topological spaces. The motion described by such systems is turbulent if the trajectories are irregular and very complicated. One of such approaches of the descriptions of such systems is in the paper of Prodi [12], where stationary turbulences appear when a nontrivial invariant ergodic measure exists. The existence of turbulent trajectories comes from the Krylov-Bogolubov theorem on the existence of invariant measures and conversely, from the individual ergodic Birkhoff theorem we get that the existence of an invariant ergodic measure implies that almost all trajectories are irregular and complicated. 
Further in [8], A. Lasota uses the above result to a linear partial differential equation of the first order. The equation depends on a certain parameter $\lambda$ that is the Reynolds number. For $\lambda$ sufficiently small $(\lambda<1)$ the solution tends to the laminar solution. For large values of $\lambda(\lambda \geq 2)$ the equation has infinitely many turbulent solutions. It is sometimes strange because usually the turbulences appear for some nonlinear partial differential equations of higher orders. In the proof presented in [8] the existence of the strictly turbulent trajectory for $\lambda \geq 2$ is proved for the subspace of functions with bounded second derivative. The existence of invariant measure on a space $V_{1}$ (see Section 3) is proved in [4] for $\lambda>1$. From [1] it follows that for $\lambda=1$ a semigroup $\left\{S_{t}\right\}$ defined here in Section 2 is stable.

The definition of the Lasota operator was created by a biological and medical motivation in the description of the creation and defferentiation of cells. The investigations were created by A. Lasota and M. Ważewska-Czyżewska in paper [10].

Then the operator was examined, for example, by Z. Brzeźniak and A.L. Dawidowicz [1], A.L. Dawidowicz [3], A.L. Dawidowicz and A. Poskrobko [5], K. Łoskot [11] as well as R. Rudnicki [13]. The Lasota operator generalizes the von Foerster operator considered, for example by A.L. Dawidowicz i N. Haribash [4], however the von Foerster operator does not differentiate the cells with respect to age.

We shall verify that the Lasota operator satisfies the approximation theorem of Wong-Zakai type. This theorem was proved by them for the one-dimensional case in paper [17]. Some generalizations of the theorem exist for the stochastic evolution eqautions, for example, in the papers of K. Twardowska [14,15]. The approximation theorem of the Wong-Zakai type is of great importance because it is a base for other theorems about the properties of the solutions of such equations, e.g. about the support of measures connected with the solutions, see the paper of K. Twardowska [16], about the invariance theorems, comparisons theorems as well as the numerical methods for solutions of such equations. The correction term appearing in the Wong-Zakai type theorems, gives better convergence of the numerical schemes. The present results were already partially published by the authors in paper [6] but Lemma 5.1 and the main theorem (Theorem 5.2) were not proved in [6].

\section{TURBULENCES AND INVARIANT MEASURES}

The most interesting results for the Lasota operator are regarding its chaotic behaviours. We shall show, for example, the results from paper [5].

Let $X$ be a topological Hausdorff space, let $\mathbb{G}$ be $\mathbb{R}$ or $\mathbb{R}_{+}$and let $S_{t}: X \rightarrow X$, $t \in \mathbb{G}$, be a family of transformations such that

$$
\begin{aligned}
S_{0} & =I \text { (identity), } \\
S_{t+s} & =S_{t} \circ S_{s}, \text { for } s \geq 0, t \in \mathbb{G} .
\end{aligned}
$$

We recall, that a family is called the semigroup of transformations if $\mathbb{G}=\mathbb{R}_{+}$and the group of transformations if $\mathbb{G}=\mathbb{R}$. We shall call the semigroup $\left\{S_{t}\right\}$ a semi-dynamical 
system if the below mapping is continuous with respect to $(t, x)$ :

$$
(t, x) \in \mathbb{R}_{+} \times X \rightarrow S_{t} x \in X .
$$

Analogously the group $\left\{S_{t}\right\}_{t \in \mathbb{R}}$ satisfying (2.1), (2.2) is called the dynamical system if the mapping

$$
(t, x) \in \mathbb{R} \times X \rightarrow S_{t} x \in X
$$

is continuous with respect to $(t, x)$.

We shall introduce the following notation for the trajectory (orbit) starting from the point $x$ and for the limit set:

$$
\begin{aligned}
& O(x)=\left\{S_{t}(x): t \geq 0\right\}, \\
& L(x)=\bigcap_{t \geq 0} \overline{O\left(S_{t}(x)\right)} .
\end{aligned}
$$

A point $x \in X$ is called periodic if there exists $t>0$ such that $S_{t}(x)=x$, so each fixed point for $S_{t}$ is periodic.

Definition 2.1. We say that the trajectory $O(x)$ is strictly turbulent if:

(i) $L(x)$ is a compact nonempty set,

(ii) $L(x)$ does not contain periodic points.

In paper [8], A. Lasota proved the following theorem.

Theorem $2.2([8])$. Assume that $\left\{S_{t}\right\}$ is a semi-dynamical system acting on a topological Hausdorff space $X$ and assume that there exists a number $r>0$ and two nonempty compact disjoint sets $A, B \subset X$ such that

$$
A \cup B \subset S_{r}(A) \cap S_{r}(B) .
$$

Then there exists a point $x_{0} \in X$ such that the trajectory $O\left(x_{0}\right)$ is strictly turbulent.

Further, from papers [5] and [8] there exists a version of Theorem 2.2 ([5]) that can be applied for some dynamical systems, too. The system described in our section 3 is an example of such a system given by the Lasota operator.

Thus we have

Theorem $2.3([8])$. Let $\left\{S_{t}\right\}$ and $\left\{T_{t}\right\}$ be semi-dynamical systems defined on compact Hausdorff topological spaces $X$ and $Y$, respectively. Let $F: X \rightarrow Y$ be a continuous mapping. Assume that for each $t \geq 0$ the following diagram commutes:

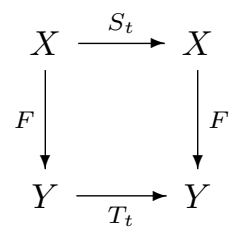


Suppose, moreover, that there exist a number $r>0$ and two nonempty closed disjoint sets $A, B \subset X$ such that

$$
A \cup B \subset T_{r}(A) \cap T_{r}(B) .
$$

Then there exists a point $x_{0} \in X$ such that the trajectory $O_{s}\left(x_{0}\right)=\left\{S_{t}\left(x_{0}\right): t \geq 0\right\}$ is strictly turbulent.

Definition 2.4. A measure $\mu$ is invariant with respect to $\left\{S_{t}\right\}$ if $\mu(E)=\mu\left(S_{t}^{-1}(E)\right)$ for every $t$ and for every borel subset $E$ of the set $X$.

Definition 2.5. A measure $\mu$ is called ergodic if for every borel set $E$, from the condition

it implies that

$$
E=S_{t}^{-1}(E) \quad \text { for } t \geq 0
$$

$$
\mu(E)(1-\mu(E))=0 .
$$

Theorem 2.6 ([9]). If $\left\{S_{t}\right\}, t \geq 0$, admits a strictly turbulent trajectory, then there exists for $\left\{S_{t}\right\}, t \geq 0$, a nontrivial ergodic invariant measure.

\section{THE LASOTA OPERATOR}

Let us consider the following linear partial differential equation of the first order

$$
\frac{\partial u}{\partial t}+x \frac{\partial u}{\partial x}=\lambda u, \quad 0 \leq t, \quad 0 \leq x<\infty
$$

with the following initial and boundary conditions

$$
\begin{aligned}
u(t, 0) & =0, \\
u(0, x) & =v(x), \quad 0 \leq x<\infty,
\end{aligned}
$$

where $\lambda>0$ is a constant.

By a solution we mean a continuous and differentiable function $u(t, x)$, for which this equation is satisfied for all $t \geq 0$ and $0 \leq x<\infty$.

Denote by $V$ the space of all continuous and differentiable functions $v: \mathbb{R}_{+} \rightarrow \mathbb{R}$ such that $v(0)=0$. We consider also the space $V_{1}$ of all continuous and differentiable functions $v:[0,1] \rightarrow \mathbb{R}$ such that $v(0)=0$. The space $V_{1}$ is normed by the norm defined by the formula

$$
\|v\|_{V_{1}}=\sup _{x \in[0,1]}\left|v^{\prime}(x)\right| .
$$

It is known, that for every $v \in V$ there exists exactly one solution to the above equation and this solution is given by the following formula:

$$
u(t, x)=e^{\lambda t} v\left(x e^{-t}\right), \quad 0 \leq x<\infty .
$$

Define the following strongly continuous semigroup of mappings that describe the time evolution of problem (3.1), (3.2):

$$
\left(S_{t}^{\lambda} v\right)(x)=e^{\lambda t} v\left(x e^{-t}\right), \quad 0 \leq x<\infty .
$$


We have the following theorem

Theorem 3.1 ([8]). If $\lambda<1$, then for each $v \in V_{1}$ we have

$$
\lim _{t \rightarrow \infty}\left\|S_{t} v\right\|_{V_{1}}=0
$$

and the unique measure invariant under $\left\{S_{t}\right\}, t \geq 0$, is concentrated on the fixed point $v \equiv 0$. If $\lambda \geq 2$, the semi-dynamical system $\left\{S_{t}\right\}, t \geq 0$, admits a strictly turbulent trajectory and, consequently, there exists for $\left\{S_{t}\right\}, t \geq 0$, a nontrivial invariant measure.

Further, we define the infinitesimal operator for the semigroup $\left\{S_{t}\right\}, t \geq 0$, as

$$
A u=\lambda u-x \frac{\partial u}{\partial x}
$$

with the domain

$$
D(A)=\left\{v \in C^{1}([0,1], \mathbb{R}): v(0)=0\right\} .
$$

Definition 3.2. We say that the strongly continuous semigroup $\left\{S_{t}\right\}, t \geq 0$, is of contraction type if there exists a constant $M \in \mathbb{R}_{+}$such that for every $t \geq 0$ :

$$
\left\|S_{t}\right\|_{V_{1}} \leq e^{M t}
$$

Now, we shall show

Proposition 3.3. The semigroup $\left\{S_{t}\right\}, t \geq 0$, is a strongly continuous semigroup of contraction type on the space $L^{2}=L^{2}([0,1], \mathbb{R})$.

Proof. We have

$$
\begin{aligned}
\left\|S_{t} v\right\|_{L^{2}}^{2} & =\int_{0}^{1}\left|S_{t} v(x)\right|^{2} d x=\int_{0}^{1} e^{2 \lambda t}\left|v\left(x e^{-t}\right)\right|^{2} d x= \\
& =\left\{\begin{array}{c}
z=x e^{-t}, \quad x=z e^{t} \\
d x=e^{t} d z
\end{array}\right\}=\int_{0}^{e^{-t}} e^{2 \lambda t}|v(z)|^{2} e^{t} d z \leq \\
& \leq e^{(2 \lambda+1) t}\|v\|_{L^{2}}^{2} .
\end{aligned}
$$

Therefore,

$$
\left\|S_{t}\right\|_{L^{2}} \leq e^{\left(\lambda+\frac{1}{2}\right) t}
$$

so $\left\{S_{t}\right\}, t \geq 0$, is the semigroup of contraction type for $M=\lambda+\frac{1}{2}$, which completes the proof.

\section{STOCHASTIC EVOLUTION EQUATIONS}

Consider the following stochastic evolution equation on the Hilbert space $H$ :

$$
\begin{aligned}
d u(t) & =A u(t) d t+B(u(t)) d w(t), \\
u(0) & =u_{0} .
\end{aligned}
$$


The equation admits additionally the perturbances by the exterior random events. It is such because the Lasota equation describes the dynamics of the population, approximately only. The error of the approximation is defined in the stochastic term that is given by an operator acting on the Wiener process.

Let $H$ and $H_{1}$ be real separable Hilbert spaces with the norms $\|\cdot\|_{H}$ and $\|\cdot\|_{H_{1}}$ and the scalar products $\langle\cdot, \cdot\rangle_{H}$ and $\langle\cdot, \cdot\rangle_{H_{1}}$, respectively. Let $\left(\Omega, \mathcal{F},(\mathcal{F})_{t \in[0, T]}, P\right)$ be a filtered probability space on which an increasing and right-continuous family $\left(\mathcal{F}_{t}\right)_{t \in[0 . T]}$ of complete sub- $\sigma$-algebras of $\mathcal{F}$ is defined. Let $L\left(H, H_{1}\right)$ denotes the space of bounded operators from $H$ to $H_{1}$. Let $L_{2}\left(H, H_{1}\right)$ denotes the space of Hilbert-Schmidt operators with the norm $\|\cdot\|_{H S}$.

Consider an $H$-valued Wiener process $w(t)$ with nuclear covariance operator $Q \in$ $L(H, H)=L(H)$.

It is known [2] that there are real-valued independent Wiener processes $\left\{w_{i}(t)\right\}_{i=0}^{\infty}$ on $[0, T]$ such that

$$
w(t)=\sum_{i=0}^{\infty} w_{i}(t) e_{i}
$$

almost surely with respect to $\omega \in \Omega$, where $\left\{e_{i}\right\}_{i=0}^{\infty}$ is the orthonormal basis of eigenvectors of $Q$, corresponding to eigenvalues $\left\{\lambda_{i}\right\}_{i=0}^{\infty}$, where $\sum_{i=0}^{\infty} \lambda_{i}<\infty$, and

$$
E\left[\Delta w_{i} \Delta w_{j}\right]=(t-s) \lambda_{i} \delta_{i j}
$$

for $\Delta w_{i}=w_{i}(t)-w_{j}(t), s<t\left(\delta_{i j}\right.$ is the Kronecker delta).

Introduce the $n$-th approximation of the Wiener process $(w(t))_{t \in[0, T]}$ :

$$
w^{n}(t)=\sum_{j=0}^{\infty} w_{j}^{n}(t) e_{j},
$$

where $0=t_{0}^{n}<\ldots<t_{n}^{n}$ and for $t_{i-1}^{n}<t \leq t_{i}^{n}$ we define

$$
w_{j}^{n}(t)=\frac{t-t_{i-1}^{n}}{t_{i}^{n}-t_{i-1}^{n}} w_{j}^{n}\left(t_{i}^{n}\right)+\frac{t_{i}^{n}-t}{t_{i}^{n}-t_{i-1}^{n}} w_{j}\left(t_{i-1}^{n}\right) .
$$

Assume:

(A1) $(u(t))_{t \in[0, T]}$ is an $H_{1}$-valued stochastic process, $A: D(A) \subset H_{1} \rightarrow H_{1}$ is the infinitesimal generator of a strongly continuous semigroup $\{S(t)\}_{t \in[0, T]}$, $B: H_{1} \rightarrow L\left(H, H_{1}\right)$ is a nonlinear operator, $\{S(t)\}_{t \in[0, T]}$ is a semigroup of contraction type;

(A2) $u_{0} \in D(A)$ is an initial random variable, square integrable, $\mathcal{F}_{0}$-measurable and with values in $H_{1}$;

(A3) there exist a constant $K>0$ and a positive definite symmetric nuclear operator $R$ which commutes with $S$, such that $P\left(R^{-1} z_{0} \in H_{1}\right)=1$ and the following conditions are satisfied:

(i)

$$
\left\|R^{-1} B\left(h_{1}\right) Q^{\frac{1}{2}}\right\|_{H S}^{2}+\left\|R^{-1} \tilde{\operatorname{tr}}\left(Q D B\left(h_{1}\right) B\left(h_{1}\right)\right)\right\|_{H_{1}}^{2} \leq K\left(1+\left\|h_{1}\right\|_{H_{1}}^{2}\right),
$$


(ii)

$$
\operatorname{tr}\left(\left(B\left(h_{1}\right)-B\left(\widetilde{h}_{1}\right)\right) Q\left(B\left(h_{1}\right)-B\left(\widetilde{h}_{1}\right)\right)^{*}\right) \leq K\left\|h_{1}-\widetilde{h}_{1}\right\|_{H_{1}}^{2}
$$

for $h_{1}, \widetilde{h}_{1} \in H_{1}$, where "*" denotes the adjoint operator,

(A4) the operator $B \in C_{b}^{1}$, i.e. is of class $C^{1}$ with bounded derivative, and this derivative is globally Lipschitzian;

(A5) the operator $D B\left(h_{1}\right) A: D(A) \subset H_{1} \rightarrow L\left(H, H_{1}\right)$ can be uniquely extended to a bounded operator from $H_{1}$ to $L\left(H, H_{1}\right)$, that is, there exists a positive constant $k$ such that for $h_{1} \in H_{1}$ we get

$$
\left\|D B\left(h_{1}\right) A h_{1}\right\|_{L\left(H, H_{1}\right)} \leq k\left\|h_{1}\right\|_{H_{1}} .
$$

Apart from (4.1) we consider the equation

$$
\begin{aligned}
d \widehat{u}(t) & =A \widehat{u}(t) d t+B(\widehat{u}(t)) d w(t)+\frac{1}{2} \widetilde{\operatorname{tr}}(Q D B(\widehat{u}(t)) B(\widehat{u}(t))) d t, \\
\widehat{u}(0) & =u_{0}
\end{aligned}
$$

where $\frac{1}{2} \widetilde{\operatorname{tr}}(Q D B(\widehat{u}(t)) B(\widehat{u}(t)))$ is the so-called correction term implying from the approximation theorem of Wong-Zakai type. It is defined in papers of K. Twardowska $[14,15]$.

We recall the definition of $\tilde{\mathrm{tr}}$ from $[14,15]$. First observe that the Fréchet derivative $D B\left(h_{1}\right) \in L\left(H_{1}, L\left(H, H_{1}\right)\right.$ for $h_{1} \in H_{1}$ and we consider the composition $D B\left(h_{1}\right) B\left(h_{1}\right) \in L\left(H, L\left(H, H_{1}\right)\right)$. We view the Fréchet derivative of $B\left(h_{1}\right)$ as $D B\left(h_{1}, h_{2}\right)$ since $h_{2} \rightarrow D B\left(h_{1}, h_{2}\right), h_{2} \in H_{1}$, is linear and belongs to $L\left(H_{1}, L\left(H, H_{1}\right)\right)$. Let $\Psi \in L\left(H, L\left(H, H_{1}\right)\right)$ and define

$$
B_{\widetilde{h}_{1}}\left(h, h^{\prime}\right)=\left(\Psi(h)\left(h^{\prime}\right), \widetilde{h}_{1}\right)_{H_{1}} \in \mathbb{R}
$$

for $h, h^{\prime} \in H$. From the Riesz theorem for the form $\Psi$ on $H$ we conclude that for every $\widetilde{h}_{1} \in H_{1}$ there exists an operator $\widetilde{\Psi}\left(\widetilde{h}_{1}\right) \in L(H)$ such that for every $h, h^{\prime} \in H$

$$
B_{\widetilde{h}_{1}}\left(h, h^{\prime}\right)=\left(\widetilde{\Psi}\left(\widetilde{h}_{1}\right)(h), h^{\prime}\right)_{H}=\left(\Psi(h)\left(h^{\prime}\right), \widetilde{h}_{1}\right)_{H_{1}} .
$$

Now, the covariance operator $Q$ has finite trace and therefore the mapping

$$
\widetilde{\xi}: \widetilde{h}_{1} \in H_{1} \rightarrow \operatorname{tr}\left(Q \widetilde{\Psi}\left(\widetilde{h}_{1}\right)\right) \in \mathbb{R}
$$

is a linear bounded functional on $H_{1}$. Therefore, using the Riesz theorem we find a unique $\widetilde{\widetilde{h}}_{1} \in H_{1}$ such that $\widetilde{\xi}\left(\widetilde{h}_{1}\right)=\left(\widetilde{\widetilde{h}}_{1}, \widetilde{h}_{1}\right)_{H_{1}}$. Define

$$
\widetilde{\widetilde{h}}_{1}=\widetilde{\operatorname{tr}}(Q \Psi)
$$

We observe that $\left(\widetilde{\widetilde{h}}_{1}, \widetilde{h}_{1}\right)_{H_{1}}$ is the trace of the operator $Q \widetilde{\Psi}\left(\widetilde{h}_{1}\right) \in L(H)$ but $\widetilde{\operatorname{tr}}(Q \Psi)$ is merely a symbol for $\widetilde{\widetilde{h}}_{1}$. 
Definition 4.1. We say that the process $(u(t))_{t \in[0, T]}$ is a mild solution to equation (4.1) if:

(i) $(u(t))_{t \in[0, T]}$ is a progressively measurable process,

(ii) $B(u(\cdot)) \in \Lambda_{T}\left(w, H, H_{1}\right)$, where

$$
\begin{aligned}
\Lambda_{T}\left(w, H, H_{1}\right)=\left\{\begin{array}{l}
\Psi:[0, T] \times \Omega \rightarrow L\left(H, H_{1}\right), \\
\Psi \text { is a progressively measurable process, }
\end{array}\right. \\
E\left[\int_{0}^{T}\left\|\Psi Q^{\frac{1}{2}}\right\|_{H S}^{2} d s\right]=\|\Psi\|_{\Lambda_{t}}^{2}= \\
\left.=\sum_{i=0}^{\infty} E\left[\int_{0}^{T}\left\|\Psi(s, \omega) e_{i}\right\|_{H_{1}}^{2} d s\right]<\infty\right\},
\end{aligned}
$$

(iii) for every $t \in[0, T]$ there exists $\Omega_{t}, \quad P\left(\Omega_{t}\right)=1$, such that equation (4.1) is satisfied for every $\omega \in \Omega_{t}$.

Consider the following sequence of approximation equations

$$
\begin{aligned}
d u^{n}(t) & =A u^{n}(t) d t+B\left(u^{n}(t)\right) d w^{n}(t), \\
u^{n}(0) & =u_{0},
\end{aligned}
$$

where $\left(w^{n}(t)\right)_{t \in[0, T]}$ is the approximation sequence of the Wiener processes given by equation (4.2).

It is known from the theory of stochastic differential equations that there exists exactly one solutions to systems (4.1), (4.3) and (4.5), respectively.

We have the following

Theorem $4.2([15])$. Assume that $\left(w^{n}(t)\right)_{t \in[0, T]}$ is the $n$-th approximation of the Wiener process $(w(t))_{t \in[0, T]}$, given by $(4.2)$. Let $\left(u^{n}(t)\right)_{t \in[0, T]}$ be the solutions of the sequence of approximation equations (4.5) and $\widehat{u}(t)$ be the solution to equation (4.3). Suppose that assumptions (A1)-(A5) are satisfied and $E\left[\left\|R^{-1} u_{0}\right\|_{H_{1}}^{2}\right]<\infty$. Then, for every $T, 0<T<\infty$ and for given $\varepsilon>0$ we get

$$
\lim _{n \rightarrow \infty} P\left(\sup _{0 \leq t \leq T}\left\|u^{n}(t, \omega)-\widehat{u}(t, \omega)\right\|_{H_{1}} \geq \varepsilon\right)=0 .
$$

\section{THE HAMMERSTEIN OPERATOR AS PERTURBATION}

Now let us take $H=H_{1}=L^{2}([0,1])$ with an orthonormal basis $\left\{e_{n}\right\}_{n=0}^{\infty}$ given by the formula

$$
e_{n}(s)=\cos \pi n s .
$$


Let $A$ be the infinitesimal generator of a semigroup for the Lasota operator. Define the integral Hammerstein operator on $H_{1}$ as the operator $B: H_{1} \rightarrow L\left(H, H_{1}\right)$ of the form:

$$
B(h)\left(e_{i}\right)(s)=\int_{0}^{1} K_{i}(s, t) f(t, h(t)) d t,
$$

where $K_{i}(s, t)=K_{i 1}(s) K_{i 2}(t), \quad K_{i 1}, K_{i 2} \geq 0$ and $K_{i 1}(s) \in C^{4}([0,1]), K_{i 2}(t) \in$ $C([0,1]), f \in C^{1}([0,1] \times \mathbb{R})$. Moreover, we assume, that:

(H1) there exist $a$ and $b>0$, such that $|f(t, x)| \leq a(t)+b|x|$

(H2) there exists $\mathcal{M}>0$, such that

$$
|f(t, x)-f(t, y)| \leq \mathcal{M}|x-y|,
$$

(H3) there exist $\mathcal{K}_{1}$, such that

$$
\int_{0}^{1} K_{i 1}(s)^{2} d s \leq \mathcal{K}_{1}
$$

and

(H4) there exist $\mathcal{K}_{2}$, such that

$$
\max _{t \in[0,1]} K_{i 2}(t) \leq \mathcal{K}_{2}
$$

for each $i=1, \ldots$.

Define the operator $R=(I-\Delta)^{-1}, \Delta=\frac{d^{2}}{d x^{2}}$. It is obvious that $R$ is a positive definite, symmetric and nuclear operator. Indeed, we have

Lemma 5.1. Let the operator $A$ defined by (3.5) and let the operator $B$ defined by (5.2) satisfy conditions $(\mathrm{H} 1)-(\mathrm{H} 4)$. Then the operators $A$ and $B$ satisfy conditions (A1)-(A5).

We shall show then that the assumptions of Theorem 4.2 are satisfied. Therefore, the approximation theorem of the Wong-Zakai type is valid for the problems with the infinitesimal generator defined by the Lasota operator and with appropriate operator $B$. Now, let us notice that for the bounded operator $M: H \rightarrow K$ and nuclear operator $N: K \rightarrow H$, where $H$ and $K$ are two separable Hilbert spaces, we have

$$
\operatorname{tr}(M N)=\operatorname{tr}(N M)
$$

This equality is obvious [7] (Ex. 5.8. p. 125).

Proof of Lemma 5.1. First, let us notice that

$$
D B_{x_{0}}(h)\left(e_{i}\right)(s)=\int_{0}^{1} K_{i}(s, t) f_{x}^{\prime}\left(t, x_{0}(t)\right) h(t) d t .
$$

Assumptions (A1), (A2) and (A4) are satisfied by our definitions and by the assumed properties of the operators [14]. 
We shall verify assumption (A3) (i). First, we shall show that there exists a constant $K>0$ and a positive-definite symmetric nuclear operator $R$, that commutes with $S$, such that $P\left(R^{-1} z_{0} \in H_{1}\right)=1$ and

$$
\left\|R^{-1} \widetilde{\operatorname{tr}}\left(Q D B\left(h_{1}\right) B\left(h_{1}\right)\right)\right\|_{H_{1}}^{2} \leq K\left(1+\left\|h_{1}\right\|_{H_{1}}^{2}\right),
$$

for $h_{1} \in H_{1}$. From (5.1) it follows, that

$$
\frac{d^{2}}{d t^{2}} e_{n}=-n^{2} \pi^{2} e_{n}
$$

Indeed,

$$
\begin{aligned}
\left\|R^{-1} \widetilde{\operatorname{tr}}\left(Q D B\left(h_{1}\right) B\left(h_{1}\right)\right)\right\|_{H_{1}}^{2} & =\sum_{n=0}^{\infty}\left|\left\langle R^{-1} \widetilde{\operatorname{tr}}\left(Q D B\left(h_{1}\right) B\left(h_{1}\right)\right), e_{n}\right\rangle_{H_{1}}\right|^{2}= \\
& =\sum_{n=0}^{\infty}\left|\left\langle\widetilde{\operatorname{tr}}\left(Q D B\left(h_{1}\right) B\left(h_{1}\right)\right),\left(1-\frac{d^{2}}{d x^{2}}\right) e_{n}\right\rangle_{H_{1}}\right|^{2}= \\
& =\widetilde{K} \sum_{n=0}^{\infty}\left|\left\langle\widetilde{\operatorname{tr}}\left(Q D B\left(h_{1}\right) B\left(h_{1}\right)\right),\left(1+n^{2} \pi^{2}\right) e_{n}\right\rangle_{H_{1}}\right|^{2} \leq \\
\leq & \widetilde{K}_{1} \sum_{n=0}^{\infty}\left[\left|\left\langle\widetilde{\operatorname{tr}}\left(Q D B\left(h_{1}\right) B\left(h_{1}\right)\right), e_{n}\right\rangle_{H_{1}}\right|^{2}+\right. \\
& \left.\quad+n^{4}\left|\left\langle\widetilde{\operatorname{tr}}\left(Q D B\left(h_{1}\right) B\left(h_{1}\right)\right), e_{n}\right\rangle_{H_{1}}\right|^{2}\right] .
\end{aligned}
$$

Now we estimate

$$
\left|\left\langle\widetilde{\operatorname{rr}}\left(Q D B\left(h_{1}\right) B\left(h_{1}\right)\right), e_{n}\right\rangle_{H_{1}}\right|^{2}=\lambda_{n}^{2}\left|\int_{0}^{1} \int_{0}^{1} K_{i}(s, t) f_{x}^{\prime}\left(t, B\left(h_{1}\right)\left(e_{i}\right)(t)\right) h_{1}(t) d t d s\right|^{2}
$$

because

$$
\left\langle e_{i}, e_{n}\right\rangle=0 \text { for } i \neq n \text {. }
$$

We have

$$
\begin{aligned}
\left|D B\left(h_{1}\right) B\left(h_{1}\right)\left(e_{i}\right)(s)\right| & =\left|\int_{0}^{1} K_{i 1}(s) \int_{0}^{1} K_{i 2}(t) f_{x}^{\prime}\left(t, B\left(h_{1}\right)\left(e_{i}\right)(t)\right) h_{1}(t) d t d s\right| \leq \\
& \left.\leq \lambda_{n} \int_{0}^{1}\left|K_{i 1}(s)\right| d s \int_{0}^{1}\left|K_{i 2}(t)\right|\left[a(t)+b\left|B\left(h_{1}\right)\left(e_{i}\right)(t)\right|\right]\right)\left|h_{1}(t)\right| d t .
\end{aligned}
$$

Now

$$
\begin{aligned}
\left|\int_{0}^{1} K_{1}(s)\left(e_{n}\right)(s) d s\right| & \leq \widetilde{K} \int_{0}^{1} K_{1}(s)\left(e_{n}\right)(s) d s=\int_{0}^{1} K(s)\left(e_{n}\right)(s) d s \leq \\
& \leq\left[\int_{0}^{1} K_{1}^{2}(s) d s \cdot \int_{0}^{1}\left|e_{n}^{2}(s)\right| d s\right]^{\frac{1}{2}} .
\end{aligned}
$$


But we have

$$
e_{n}(s)=\cos \pi n s
$$

so

$$
\begin{aligned}
\int_{0}^{1} K_{1}(s)\left(e_{n}\right)(s) d s & =\left.\frac{1}{\pi n} K_{1}(s) \sin \pi n s\right|_{0} ^{1}-\frac{1}{\pi n} \int_{0}^{1} K_{1}^{\prime}(s) \sin \pi n s d s= \\
& =-\frac{1}{\pi n} \int_{0}^{1} K_{1}^{\prime}(s) \sin \pi n s d s= \\
& =\left.\frac{1}{\pi^{2} n^{2}} K_{1}^{\prime}(s) \cos \pi n s\right|_{0} ^{1}+\frac{1}{\pi^{2} n^{2}} \int_{0}^{1} K_{1}^{\prime \prime}(s) \cos \pi n s d s .
\end{aligned}
$$

Further

$$
\begin{aligned}
\int_{0}^{1} K_{1}(s) \cos \pi n s d s & =\frac{1}{\pi n} \int_{0}^{1} K_{1}(s)(\sin \pi n s)^{\prime} d s= \\
& =\frac{1}{\pi n}\left[\left.K_{1}(s) \sin \pi n s\right|_{0} ^{1}-\int_{0}^{1} K_{1}^{\prime}(s) \sin \pi n s d s\right]= \\
& =\frac{1}{\pi^{2} n^{2}} \int_{0}^{1} K_{1}^{\prime}(s)(\cos \pi n s)^{\prime} d s= \\
& =\frac{1}{\pi^{2} n^{2}}\left[\left.K_{1}(s) \cos \pi n s\right|_{0} ^{1}-\int_{0}^{1} K_{1}^{\prime \prime}(s) \cos \pi n s d s\right] .
\end{aligned}
$$

We have

$$
\left|\int_{0}^{1} K_{1}(s) e_{n}(s) d s\right|^{2} \leq \frac{c}{n^{4}}
$$

Now we have

$$
\left\langle\widetilde{\operatorname{tr}}\left(Q D B\left(h_{1}\right) B\left(h_{1}\right)\right), e_{n}\right\rangle_{H_{1}}=D B\left(h_{1}\right) B\left(h_{1}\right)\left(e_{n}\right)=\sum_{j=0}^{\infty}\left[D B\left(h_{1}\right) B\left(h_{1}\right)\left(e_{j}\right)\right]\left(\lambda_{j} e_{j}\right) .
$$

Moreover,

$$
\begin{aligned}
e_{n}(t) & =e^{2 \pi i n t}, \\
-\frac{d^{2}}{d t^{2}} e_{n}(t) & =n^{2} e_{n}, \\
-\frac{d^{2}}{d x^{2}}(\cos 2 \pi n x) & =n^{2}(\cos 2 \pi n x) .
\end{aligned}
$$


Now

$$
\begin{aligned}
\left|\left\langle\widetilde{\operatorname{tr}}\left(Q D B\left(h_{1}\right) B\left(h_{1}\right)\right), e_{n}\right\rangle_{H_{1}}\right|^{2} & =\lambda_{n}^{2}\left|\int_{0}^{1} K_{n}(s, t) f_{x}^{\prime}\left(t, B\left(h_{1}\right)\left(e_{i}\right)(t)\right) h_{1}(t) d t\right|^{2} \leq \\
& \leq c \lambda_{n}^{2}\left\|h_{1}\right\|_{H_{1}}^{2} \leq \frac{c \widetilde{K}^{2}}{n^{4}}\left\|h_{1}\right\|_{H_{1}}^{2}
\end{aligned}
$$

under assumptions $f_{x}^{\prime} \leq a(t)+b|x|, K_{n}$ is bounded by $c$ independently of $n, \lambda_{n} \leq \frac{\widetilde{K}}{n^{2}}$.

Further, we shall show that there exists a constant $K>0$ and a positive-definite symmetric nuclear operator $R$, that commutes with $S$, such that $P\left(R^{-1} z_{0} \in H_{1}\right)=1$ and

$$
\left\|R^{-1} B\left(h_{1}\right) Q^{\frac{1}{2}}\right\|_{H S}^{2}+\left\|R^{-1} \tilde{\operatorname{tr}}\left(Q D B\left(h_{1}\right) B\left(h_{1}\right)\right)\right\|_{H_{1}}^{2} \leq K\left(1+\left\|h_{1}\right\|_{H_{1}}^{2}\right),
$$

for $h_{1} \in H_{1}$. Indeed, we have

$$
\begin{aligned}
\sum_{n \geq 1}\left|R^{-1} B\left(h_{1}\right) Q^{\frac{1}{2}} e_{n}\right|^{2} & =\sum_{n \geq 1}\left|R^{-1} B\left(h_{1}\right) \lambda^{\frac{1}{2}} e_{n}\right|^{2}= \\
& =\sum_{n \geq 1} \lambda_{n}\left\|R^{-1} B\left(h_{1}\right) e_{n}\right\|^{2} \leq \sum_{n \geq 1} \lambda_{n} \widetilde{K}\left(1+\left\|h_{1}\right\|^{2}\right) .
\end{aligned}
$$

We have

$$
\begin{aligned}
R^{-1} B\left(h_{1}\right) e_{n} & =(I-\Delta) \int_{0}^{1} K_{1}(s, t) f\left(t, h_{1}(t)\right) d t= \\
& =\left[\int_{0}^{1} K_{1}(s, t) f\left(t, h_{1}\right) d t-\int_{0}^{1} K_{1}^{\prime \prime}(s, t) f\left(t, h_{1}(t)\right) d t\right]= \\
& =\int_{0}^{1} L(s, t) f\left(t, h_{1}(t)\right) d t .
\end{aligned}
$$

Moreover,

$$
\begin{aligned}
\left\|R^{-1} B\left(h_{1}\right) e_{n}\right\|^{2} & =\int_{0}^{1}\left[\int_{0}^{1} L(s, t) f\left(t, h_{1}(t)\right) d t\right]^{2} d s \leq \\
& \leq \int_{0}^{1}\left[\int_{0}^{1} L(s, t)^{2} d t\right] \cdot\left[\int_{0}^{1} f\left(t, h_{1}(t)\right)^{2} d t\right] d s \leq \\
& \leq \int_{0}^{1}\left[\int_{0}^{1} L(s, t)^{2} d t\right] \cdot\left[\int_{0}^{1}\left(a(t)+b\left|h_{1}(t)\right|^{2}\right) d t\right] d s \leq \\
& \leq \widehat{K}\left(1+\left\|h_{1}\right\|^{2}\right) \int_{0}^{1} \int_{0}^{1} L(s, t)^{2} d t d s .
\end{aligned}
$$


From (5.4) and (5.5) we get (A3) (i).

Now we shall verify (A3) (ii).

From (5.3) it follows, that the condition (A3) (ii) is equivalent to

$$
\operatorname{tr}\left(Q\left(B\left(h_{1}\right)-B\left(\widetilde{h}_{1}\right)\right)^{*}\left(B\left(h_{1}\right)-B\left(\widetilde{h}_{1}\right)\right)\right) \leq K\left\|h_{1}-\widetilde{h}_{1}\right\|_{H_{1}}^{2} .
$$

Notice, that

$$
\begin{aligned}
& \operatorname{tr}\left(Q\left(B\left(h_{1}\right)-B\left(\widetilde{h}_{1}\right)\right)^{*}\left(B\left(h_{1}\right)-B\left(\widetilde{h}_{1}\right)\right)\right) \leq \\
& \leq\left(\sum_{n=1}^{\infty} \lambda_{n}\right)\left\|\left(B\left(h_{1}\right)-B\left(\widetilde{h}_{1}\right)\right)^{*}\left(B\left(h_{1}\right)-B\left(\widetilde{h}_{1}\right)\right)\right\| \leq \\
& \leq\left(\sum_{n=1}^{\infty} \lambda_{n}\right)\left\|B\left(h_{1}\right)-B\left(\widetilde{h}_{1}\right)\right\|^{2} .
\end{aligned}
$$

Moreover

$$
\begin{aligned}
\left|B\left(h_{1}\right)\left(e_{i}\right)(s)-B\left(\widetilde{h_{1}}\right)\left(e_{i}\right)(s)\right| & =\left|\int_{0}^{1} K_{i}(s, t)\left(f\left(t, h_{1}(t)\right)-f\left(t, \widetilde{h_{1}}(t)\right)\right) d t\right| \leq \\
& \leq \int_{0}^{1}\left|K_{i}(s, t)\right| \mathcal{M}\left|h_{1}(t)-\widetilde{h_{1}}(t)\right| d t,
\end{aligned}
$$

where $\mathcal{M}$ is defined by (H2). From the Schwarz inequality it follows, that

$$
\begin{aligned}
\left\|B\left(h_{1}\right)\left(e_{i}\right)-B\left(\widetilde{h_{1}}\right)\left(e_{i}\right)\right\|^{2} & \leq\left(\int_{0}^{1} K_{i 1}^{2}(s) d s\right)\left(\int_{0}^{1}\left(K_{i 2}(t)\left(h_{1}(t)-\widetilde{h_{1}}(t)\right)\right)^{2} d t\right) \leq \\
& \leq \mathcal{K}_{1} \mathcal{K}_{2}\left\|h_{1}-\widetilde{h_{1}}\right\|^{2} .
\end{aligned}
$$

Hence

$$
\begin{aligned}
\left\|\left(B\left(h_{1}\right)-B\left(\widetilde{h_{1}}\right)\right)(x)\right\|^{2} & =\sum_{n=1}^{\infty}\left\langle x, e_{n}\right\rangle^{2}\left\|\left(B\left(h_{1}\right)-B\left(\widetilde{h_{1}}\right)\right)\left(e_{n}\right)\right\|^{2} \leq \\
& \leq \sum_{n=1}^{\infty}\left\langle x, e_{n}\right\rangle^{2} \mathcal{K}_{1} \mathcal{K}_{2}\left\|h_{1}-\widetilde{h_{1}}\right\|^{2}=\mathcal{K}_{1} \mathcal{K}_{2}\left\|h_{1}-\widetilde{h_{1}}\right\|^{2}\|x\|^{2} .
\end{aligned}
$$

Hence

$$
\left\|\left(B\left(h_{1}\right)-B\left(\widetilde{h_{1}}\right)\right)\right\|^{2} \leq \mathcal{K}_{1} \mathcal{K}_{2}\left\|h_{1}-\widetilde{h_{1}}\right\|^{2}
$$

and finally

$$
\operatorname{tr}\left(Q\left(B\left(h_{1}\right)-B\left(\widetilde{h}_{1}\right)\right)^{*}\left(B\left(h_{1}\right)-B\left(\widetilde{h}_{1}\right)\right)\right) \leq\left(\sum_{n=1}^{\infty} \lambda_{n}\right) \mathcal{K}_{1} \mathcal{K}_{2}\left\|h_{1}-\widetilde{h_{1}}\right\|^{2} .
$$


The last inequality guarantees, that the operator $B$ satisfies condition (A3) (ii).

Now we shall verify assumption (A5). We have from the definition of the operator $A$ :

$$
\begin{aligned}
D B\left(h_{1}\right) A h_{1}\left(e_{i}\right)(s) & =\lambda \int_{0}^{1} K_{i}(s, t) f_{x}^{\prime}\left(t, h_{1}(t)\right) h_{1}(t) d t-\int_{0}^{1} K_{i}(s, t) f_{x}^{\prime}\left(t, h_{1}(t)\right) t h_{1}^{\prime}(t) d t \\
& =I_{1}-I_{2} .
\end{aligned}
$$

We estimate $I_{2}$. We get by integrating by parts:

$$
\begin{aligned}
I_{2}= & \int_{0}^{1}\left[K_{i}(s, t) f_{x}^{\prime}\left(t, h_{1}(t)\right) t\right] h_{1}^{\prime}(t) d t= \\
= & K_{i}(s, 1) f_{x}^{\prime}\left(1, h_{1}(1)\right) 1 h_{1}(1)-K_{i}(s, 0) f_{x}^{\prime}\left(0, h_{1}(0)\right) 0 h_{1}(0)- \\
& -\int_{0}^{1}\left[K_{i}(s, t) f_{x}^{\prime}\left(t, h_{1}(t)\right) t\right]_{t}^{\prime} h_{1}(t) d t= \\
= & K_{i}(s, 1) f_{x}^{\prime}\left(1, h_{1}(1)\right) h_{1}(1)-\int_{0}^{1} K_{i t}^{\prime}(s, t) f_{x}^{\prime}\left(t, h_{1}(t)\right) t h_{1}(t) d t- \\
& -\int_{0}^{1} K_{i}(s, t) f_{x t}^{\prime \prime}\left(t, h_{1}(t)\right) t h_{1}(t) d t-\int_{0}^{1} K_{i}(s, t) f_{x x}^{\prime \prime}\left(t, h_{1}(t)\right) h_{1}^{\prime}(t) t h_{1}(t) d t- \\
& -\int_{0}^{1} K_{i}(s, t) f_{x}^{\prime}\left(t, h_{1}(t)\right) t h_{1}(t) d t .
\end{aligned}
$$

We write

$$
I_{2}=I_{21}-I_{22}-I_{23}-I_{24}-I_{25}=I_{21}-I_{22}-I_{23}-\alpha I_{2}-I_{25}
$$

The last equation may be written in the form

$$
(1+\alpha) I_{2}=I_{21}-I_{22}-I_{23}-I_{25}
$$

We see that under a suitable definition of the function $f(t, x)$, for example, to get $f_{x}^{\prime}\left(t, h_{1}(t)\right)=\alpha f_{x x}^{\prime \prime}\left(t, h_{1}(t)\right) h_{1}^{\prime}(t), \alpha \geq 0$, we have the situation that we can add the 
expressions $I_{2}$ and $I_{24}$. Then the derivatives $h_{1}^{\prime}(t)$ disappear. Thus we obtain

$$
\begin{aligned}
D B\left(h_{1}\right) A h_{1}\left(e_{i}\right)(s)= & \lambda \int_{0}^{1} K_{i}(s, t) f_{x}^{\prime}\left(t, h_{1}(t)\right) h_{1}(t) d t- \\
& -(1-\alpha) \int_{0}^{1} K_{i}(s, t) f_{x}^{\prime}\left(t, h_{1}(t)\right) t h_{1}^{\prime}(t) d t= \\
= & \lambda \int_{0}^{1} K_{i}(s, t) f_{x}^{\prime}\left(t, h_{1}(t)\right) h_{1}(t) d t-\int_{0}^{1} K_{i}(s, t) f_{x}^{\prime}\left(t, h_{1}(t)\right) t h_{1}^{\prime}(t) d t- \\
& -K_{i}(s, 1) f_{x}^{\prime}\left(1, h_{1}(1)\right) h_{1}(1)+\int_{0}^{1} K_{i t}^{\prime}(s, t) f_{x}^{\prime}\left(t, h_{1}(t)\right) t h_{1}(t) d t+ \\
& +\int_{0}^{1} K_{i}(s, t) f_{x t}^{\prime \prime}\left(t, h_{1}(t)\right) t h_{1}(t) d t+\int_{0}^{1} K_{i}(s, t) f_{x}^{\prime}\left(t, h_{1}(t)\right) t h_{1}(t) d t,
\end{aligned}
$$

and from this we get that $\left\|D B\left(h_{1}\right) A h_{1}\right\|_{L\left(H, H_{1}\right)} \leq k\left\|h_{1}\right\|_{H_{1}}$.

We have proved

Theorem 5.2. Assume that $\left(w^{n}(t)\right)_{t \in[0, T]}$ is the $n$-th approximation of the Wiener process $(w(t))_{t \in[0, T]}$, given by (4.2). Let $\left(u^{n}(t)\right)_{t \in[0, T]}$ be the solutions of the sequence of approximation equations (4.5) and $\widehat{u}(t)$ be the solution to equation (4.3) with the operator A given by (3.5) and the operator $B$ given by (5.2). Suppose that assumptions (H1)-(H4) are satisfied and $E\left[\left\|R^{-1} u_{0}\right\|_{H_{1}}^{2}<\infty\right.$. Then, for every $T, 0<T<\infty$ and for given $\varepsilon>0$ we get (4.6).

\section{REFERENCES}

[1] Z. Brzeźniak, A.L. Dawidowicz, On periodic solutions of the Lasota equation, Semigroup Forum 78 (2009), 118-137.

[2] R.F. Curtain, A.J. Pitchard, Infinite dimensional linear systems theory, Springer, Berlin, 1978.

[3] A.L. Dawidowicz, On the existence of an invariant measure for the dynamical system generated by partial differential equation, Ann. Polon. Math. XLI (1983), 129-137.

[4] A.L. Dawidowicz, N. Haribash, On the periodic solutions of von Foerster type equation, Univ. Iagel. Acta Math. 37 (1999), 321-324.

[5] A.L. Dawidowicz, A. Poskrobko, On asymptotic behaviour of the dynamical systems generated by von Foerster-Lasota equations, Control and Cybernet. 35 (2006) 4, 803-813.

[6] A.L. Dawidowicz, K. Twardowska, On an approximation theorem of Wong-Zakai type for the Lasota operator, Mat. Stosow. 8 (2007) 49, 56-65 [in Polish]. 
[7] A. Deitmar, S. Echterhoff, Principles of harmonic analysis, Springer, 2009.

[8] A. Lasota, Invariant measures and a linear model of turbulence, Rendiconti del Seminario Matematica dell'Universita di Padova 61 (1979), 39-48.

[9] A. Lasota, J. Yorke, On the existence of invariant measures for transformations with strictly turbulent trajectories, Bull. Pol. Acad. Sci., Sér. Sci. Math. Astronom. Phys. 25 (1977), 233-238.

[10] A. Lasota, M. Ważewska-Czyżewska, Mathematical problems of the dynamics of a system of red blood cells, Mat. Stosow. 6 (1976), 23-40 [in Polish].

[11] K. Łoskot, Turbulent solutions of first order partial differential equation, J. Differential Equations 58 (1985) 1, 1-14.

[12] G. Prodi, Teoremi ergodici per le equazioni della idrodinamica, C.I.M.E., Roma, 1960.

[13] R. Rudnicki, Invariant measures for the flow of a first order partial differential equation, Ergodic Theory Dynam. Systems 5 (1985) 3, 437-443.

[14] K. Twardowska, An approximation theorem of Wong-Zakai type for nonlinear stochastic partial differential equations, Stoch. Anal. Appl. 13 (1995) 5, 601-626.

[15] K. Twardowska, Approximation theorems of Wong-Zakai type for stochastic differential equations in infinite dimensions, Dissertationes Math., Polska Akademia Nauk, Instytut Matematyczny, vol. 325, Warszawa, 1993, 1-53.

[16] K. Twardowska, On support theorems for stochastic nonlinear partial differential equations [in:] Stochastic Differential and Difference Equations, I. Csiszár and Gy. Michaletzky eds., Birkhäuser, Boston, 1997, 309-317.

[17] E. Wong, M. Zakai, On the convergence of ordinary integrals to stochastic integrals, Ann. Math. Statist. 36 (1965), 1560-1564.

Antoni Leon Dawidowicz

Antoni.Leon.Dawidowicz@im.uj.edu.pl

Jagellonian University

Institute of Mathematics

ul. Łojasiewicza 6, 30-348 Cracow, Poland

Krystyna Twardowska

krystyna_twardowska@sggw.pl

Warsaw University of Life Sciences - SGGW

Faculty of Applied Informatics and Mathematics

ul. Nowoursynowska 159, 02-776 Warsaw, Poland

Received: January 8, 2010.

Accepted: March 16, 2010. 